\title{
Application of Titanium Dioxide-Graphene Composite Material for Photocatalytic Degradation of Alkylphenols
}

\author{
Chanbasha Basheer ${ }^{1,2}$ \\ ${ }^{1}$ Department of Chemistry and Center of Research Excellence in Nanotechnology, King Fahd University of Petroleum and Minerals, \\ KFUPM Box 1509, Dhahran 31261, Saudi Arabia \\ ${ }^{2}$ Department of Chemistry, National University of Singapore, 3 Science Drive 3, Singapore 117543
}

Correspondence should be addressed to Chanbasha Basheer; cbasheer@kfupm.edu.sa

Received 13 May 2012; Revised 26 June 2012; Accepted 28 June 2012

Academic Editor: Zhongfang Chen

Copyright (c) 2013 Chanbasha Basheer. This is an open access article distributed under the Creative Commons Attribution License, which permits unrestricted use, distribution, and reproduction in any medium, provided the original work is properly cited.

\begin{abstract}
Titanium dioxide-graphene $\left(\mathrm{TiO}_{2}-\mathrm{G}\right)$ composite was used for the photodegradation of alkylphenols in wastewater samples. The $\mathrm{TiO}_{2}-\mathrm{G}$ composites were prepared via sonochemical and calcination methods. The synthesized composite was characterized by Xray diffraction (XRD), infrared spectroscopy (IR), scanning electron microscopy (SEM), transmission electron microscopy (TEM), energy dispersive X-ray analysis (EDX), and fluorescence spectroscopy. The photocatalytic efficiency was evaluated by studying the degradation profiles of alkylphenols using gas chromatography-flame ionization detector (GC-FID). It was found that the synthesized $\mathrm{TiO}_{2}-\mathrm{G}$ composites exhibit enhanced photocatalytic efficiencies as compared to pristine $\mathrm{TiO}_{2}$. The presence of graphene not only provides a large surface area support for the $\mathrm{TiO}_{2}$ photocatalyst, but also stabilizes charge separation by trapping electrons transferred from $\mathrm{TiO}_{2}$, thereby hindering charge transfer and enhancing its photocatalytic efficiency.
\end{abstract}

\section{Introduction}

Alkylphenol ethoxylates (APEs) are a class of nonionic surfactants that have been used extensively as detergents, emulsifiers, and dispersing agents [1]. It has been reported that during the biodegradation of APEs in conventional wastewater treatment plants (WWTPs), alkylphenols (APs) like octylphenol (OP) and nonylphenol (NP) are produced as persistent metabolites which possess higher toxicity and estrogenic activity and have greater tendency towards bioaccumulation [2]. With widespread use of these APs and poor efficiency of biodegradation, it is difficult to remove completely from wastewater treatment plants. NP is listed as a priority pollutant in the Water Framework Directive (WFD) [3], and studies have shown that trace levels of OP and NP can exert estrogenic effects on aquatic organisms and mammals [4]. The concentrations of APs detected in wastewater effluents were in the range of $0.01-15 \mu \mathrm{gL}^{-1}$ [1] while the proposed environmental quality standard (EQS) of the WFD for nonylphenol and octylphenol in surface waters is $0.3 \mu \mathrm{gL}^{-1}$ and $0.1 \mu \mathrm{gL}^{-1}$, respectively [5].
Advanced oxidation process (AOP) involving $\mathrm{UV} / \mathrm{TiO}_{2}$ is a promising remediation technique for these phenolic endocrine-disrupting compounds since there is usually UV disinfection step in wastewater treatment plants and it is cheap. Titania is the most common semiconductor photocatalyst used due to its strong oxidizing power, nontoxicity, and long-term photostability [6]. In addition, studies have shown that complete mineralization of alkylphenols to $\mathrm{CO}_{2}$ and $\mathrm{H}_{2} \mathrm{O}$ can be achieved via the use of $\mathrm{TiO}_{2}$ [7]. However, it suffers from the major drawback of low photocatalytic efficiency due to the high rate of electron/hole pair recombination [8]. Thus the development of new materials for modifying $\mathrm{TiO}_{2}$ is needed to increase the photocatalytic activity for its practical applications in WWTPs.

When the $\mathrm{TiO}_{2}$ photocatalyst is irradiated with energy equal to or greater than the bandgap energy $(3.2 \mathrm{eV})$, the electrons move to the conduction band $(\mathrm{CB})$ to generate positive holes in the valence band (VB) [10]. The positive holes can react with adsorbed $\mathrm{H}_{2} \mathrm{O}$ to form hydroxyl radicals while the electrons react with $\mathrm{O}_{2}$ to form superoxide radicals. Being highly reactive, the $\mathrm{OH}$ and $\mathrm{O}_{2}$ radicals can oxidize 
the pollutants in solution or react with adsorbed pollutants. Otherwise, these electron and hole pairs can recombine. The high rate of electron and hole pair recombination thus restricts the efficiency of $\mathrm{TiO}_{2}$ photocatalysis. Many efforts are focused on enhancing the photoactivity of $\mathrm{TiO}_{2}$, and some of these include increasing the adsorption abilities of the photocatalyst surface by adding a coadsorbent [7] and modification of $\mathrm{TiO}_{2}$ with metal or semiconductor to improve the separation between free carriers [11].

Graphene is a flat monolayer of carbon atoms tightly packed in a two-dimensional honeycomb lattice, which has received tremendous attention over the years due to its extraordinary electronic, thermal, and mechanical properties [12]. It is a 0 ev bandgap semiconductor [13] with high mobility of charge carriers [14], high specific area $\left(\sim 2600 \mathrm{~m}^{2} \mathrm{~g}^{-1}\right)$ [15], and a high adsorption capacity. Given the properties of graphene, the presence of graphene can enhance the photocatalytic efficiency of $\mathrm{TiO}_{2}$ [16-19]. Since cost is an important consideration for practical applications in WWTPs, one added advantage of using $\mathrm{TiO}_{2}$-graphene composites as photocatalyst is that bulk production of graphene sheets can be produced from the inexpensive and abundant graphite at low costs [9].

In this study, $\mathrm{TiO}_{2}$-graphene composite was synthesized and characterized. The photocatalytic efficiencies for the degradation of 4-n-heptylphenol, 4-n-octylphenol, and 4nonylphenol of the synthesized composites were compared with $\mathrm{TiO}_{2}$ using GC-FID. The degradation profiles of these APs were studied because they are the reported byproducts of the widely used commercial formulations of APEs.

\section{Experimental}

2.1. Chemicals and Reagents. The acid-functionalized graphite oxide was obtained from Associate Professor Loh Kian Ping's research group at the National University of Singapore and was synthesized via Hummer's method [20]. Tetrabutyl orthotitanate (purity $\geq 97 \%$ ) was purchased from Fluka (Buchs, Switzerland), and hydrazine (35 weight \% solution in water) was obtained from Sigma-Aldrich (Milwaukee, WI, USA). Technical ethanol was obtained from Riverbank Chemicals Pte Ltd (Singapore). The commercial photocatalyst, $\mathrm{TiO}_{2}$ P25 (Degussa AG, Germany) with primary particle diameter of $21 \mathrm{~nm}$ and specific surface area of $50 \pm 15 \mathrm{~m}^{2} \mathrm{~g}^{-1}$, was used for comparison purposes. The following chemical standards (purity $\geq 98 \%$ ) were obtained from Wako Chemicals (Tokyo, Japan): 4-n-heptylphenol, 4-n-octylphenol, and 4-nonylphenol (mixture of NP isomers). The derivatization agent bis(trimethylsilyl)trifluoroacetamide (BSTFA) (purity > 98\%) was obtained from Merck (Darmstadt, Germany). Ultrapure water was prepared on a Milli-Q water system (Milford, MA, USA). For the HPLC-grade organic solvents, acetonitrile was obtained from Lab-Scan Analytical Sciences (Bangkok, Thailand), and hexane was obtained from Fischer Scientific (Loughborough, UK). Stock standard solutions of the individual standards were prepared in acetonitrile at $1000 \mathrm{mgL}^{-1}$ and stored at $4^{\circ} \mathrm{C}$. Aqueous solutions $(200 \mathrm{~mL})$ containing $5 \mathrm{mg} / \mathrm{L}$ mixtures of the analytes were prepared using ultrapure water.

2.2. Materials. Photodegradation was initiated via the use of a $15 \mathrm{~W}$ germicidal mercury lamp with a working wavelength of $254 \mathrm{~nm}$ from Ster-L-Ray, Atlantic Ultraviolet Corp. (Hauppauge, NY, USA). A jacketed Pyrex glass column (310 mm height and $70 \mathrm{~mm}$ inner diameter) was bought from UFO Labglass (S) Pte Ltd and was used as the photoreactor.

2.3. Synthesis of $\mathrm{TiO}_{2}$-Graphene Photocatalyst. The graphene sheets were combined with $\mathrm{TiO}_{2}$ nanoparticles through sonochemical and calcination methods [21]. Firstly, graphite oxide was sonicated in technical ethanol $(20 \mathrm{~mL})$ for 15 minutes to disperse them well and form graphene oxide solution (GO). Tetrabutyl orthotitanate (TBOT) was used as the titanium precursor, and it was added to the GO solution. Next, the mixture was sonicated for 30 minutes to improve the interaction between these chemicals. Milli$Q$ water $(80 \mathrm{~mL})$ was added, and the sol sample formed by hydrolysis was treated with ultrasonic irradiation in an ultrasonic cleaning bath (Branson 1210-USA). The sol sample was then aged at $25^{\circ} \mathrm{C}$ for 20 hours to further hydrolyze TBOT and form monodispersed $\mathrm{TiO}_{2}$ nanoparticles. The samples were dried in a $100^{\circ} \mathrm{C}$ oven. In order to reduce the GO to graphene $(\mathrm{G})$, hydrazine solution $(5 \mathrm{~mL})$ was added to the dried sample and it was stirred for 24 hours. The reduced sample, $\mathrm{TiO}_{2}$-graphene $\left(\mathrm{TiO}_{2}-\mathrm{G}\right)$, was then washed with ultrapure water and dried in a dessicator. Finally, in order to convert the amorphous $\mathrm{TiO}_{2}$ to crystalline $\mathrm{TiO}_{2}$ which possesses higher photocatalytic activity, the mixtures were calcined under nitrogen atmosphere at $400^{\circ} \mathrm{C}$ for 1 hour. $\mathrm{TiO}_{2}-\mathrm{G}$ composites with different mass ratios of titanium and carbon were synthesized $(1: 0.1$ and $1: 0.2)$. The preparation process of $\mathrm{TiO}_{2}$ was similar to that of $\mathrm{TiO}_{2}-\mathrm{G}$, except that the addition of $\mathrm{GO}$ and reduction with hydrazine steps were skipped.

2.4. Characterization Techniques. Powder X-ray diffraction (XRD) was used for phase identification and to estimate the crystallite size of the anatase nanoparticles. The XRD spectra were obtained at room temperature with a Siemens X-ray diffractometer (Model: B5005) using $\mathrm{Cu} \mathrm{K \alpha}$ radiation $(\lambda=$ $1.5406 \AA$ '), and data were collected from $2 \theta=20^{\circ}$ to $80^{\circ}$ at a step size of $0.020^{\circ}$ s. FTIR spectra of $\mathrm{TiO}_{2}, \mathrm{GO}$, and $\mathrm{TiO}_{2}$ graphene were obtained via a Varian 3100 FT-IR spectrophotometer using $\mathrm{KBr}$ pellets. Transmission electron microscopy (TEM) images and selected area electron diffraction (SAED) of the synthesized composite were performed via a JEOL JEM 3010 electron microscope to observe the surface morphology of the $\mathrm{TiO}_{2}-\mathrm{G}$ composite. EDX analysis was recorded on a JEOL JED-2300 scanning electron microscope to identify the elemental composition. The charge recombinations of $\mathrm{TiO}_{2}-\mathrm{G}$ and $\mathrm{TiO}_{2}$ were compared by obtaining the fluorescence emission spectra. Fluorescence spectroscopy analysis was carried out at room temperature via a Perkin Elmer Luminescence spectrometer (LS 55) equipped with a powder 
holder accessory, and the excitation wavelength was set at $300 \mathrm{~nm}$.

2.5. Study of Photodegradation Profiles. The photocatalytic efficiencies of the synthesized composites were evaluated by studying the degradation profiles of alkylphenols in aqueous media under Ultraviolet $C(\lambda=254 \mathrm{~nm})$ irradiation. A batch reactor, which was housed in a black box, was used for the photocatalytic experiments, and the schematic diagram is shown in Figure 1. The solution was continuously stirred in order to obtain a homogeneous solution. Cooling water was circulated in the outer jacket in order to keep the reaction temperature constant. In each photodegradation test, $20 \mathrm{mg}$ of photocatalyst was dispersed in $200 \mathrm{~mL}$ solution containing $5 \mathrm{ppm}$ mixture, and the amount of suspended photocatalyst was kept at $0.1 \mathrm{~g} / \mathrm{L}$. To allow the adsorption-desorption equilibrium of alkylphenols on the photocatalyst, the suspension was stirred for 5 minutes under dark conditions. Prior to turning on the UV lamp, the concentration of the solution was determined, which was considered as the initial concentration $\left(C_{0}\right)$.

2.6. Analytical Procedure. In order to monitor the variation of analyte concentrations with time and obtain the degradation profiles, sample aliquots were withdrawn from the photoreactor at 45-minute intervals (over a period of 3 hours) and filtered through a $0.2 \mu \mathrm{m}$ syringe filter to remove the photocatalyst particles before analyses. Liquidliquid extraction with previously optimized conditions [1] was used for study the degradation profile; briefly, a $5 \mathrm{~mL}$ of sample was adjusted to $\mathrm{pH} 2$ using $1 \mathrm{M} \mathrm{HCl}$ in a $10 \mathrm{~mL}$ centrifuge tube and extracted twice with $200 \mu \mathrm{L}$ of dichloromethane. To remove trace amounts of water, anhydrous sodium sulphate was added to the organic layer and $100 \mu \mathrm{L}$ of bis(trimethylsilyl)trifluoroacetamide (BSTFA) was added. The mixture was kept in a $60^{\circ} \mathrm{C}$ water bath for $20 \mathrm{~min}$ to complete the derivatization. From this, $2 \mu \mathrm{L}$ was injected to a GC-FID for analysis.

2.7. GC-FID Analysis. GC analyses were performed via an Agilent 7890A GC-FID system with a 7683B autosampler. The GC was equipped with a DB-5 fused silica capillary column, $30 \mathrm{~m} \times 0.25 \mathrm{~mm}$ i.d. $\times 0.25 \mu \mathrm{m}$ film thickness (J\&W Scientific, Folsom, CA, USA). Helium was used as the carrier gas at a flow rate of $1 \mathrm{mLmin}^{-1} .2 \mu \mathrm{L}$ of the derivatized sample was injected into GC-FID under the splitless mode. The injection temperature was set at $300^{\circ} \mathrm{C}$ and the temperature programming was as follows: $60^{\circ} \mathrm{C}$ (held for $2 \mathrm{~min}$ ), $20^{\circ} \mathrm{C} / \mathrm{min}$ to $180^{\circ} \mathrm{C}$, and $5^{\circ} \mathrm{Cmin}^{-1}$ to $220^{\circ} \mathrm{C}$ (held for 8 minutes).

\section{Results and Discussion}

3.1. XRD Characterization. Although there are three crystalline forms of $\mathrm{TiO}_{2}$ (anatase, brookite, and rutile), the anatase form of titania is reported to give the best combination of photoactivity and photostability [22]. The XRD spectra of the synthesized $\mathrm{TiO}_{2}$ and $\mathrm{TiO}_{2}$-graphene are shown in Figure 2. The peaks at $2 \theta=25.56^{\circ}, 38.34^{\circ}, 48.26^{\circ}, 54.48^{\circ}$,

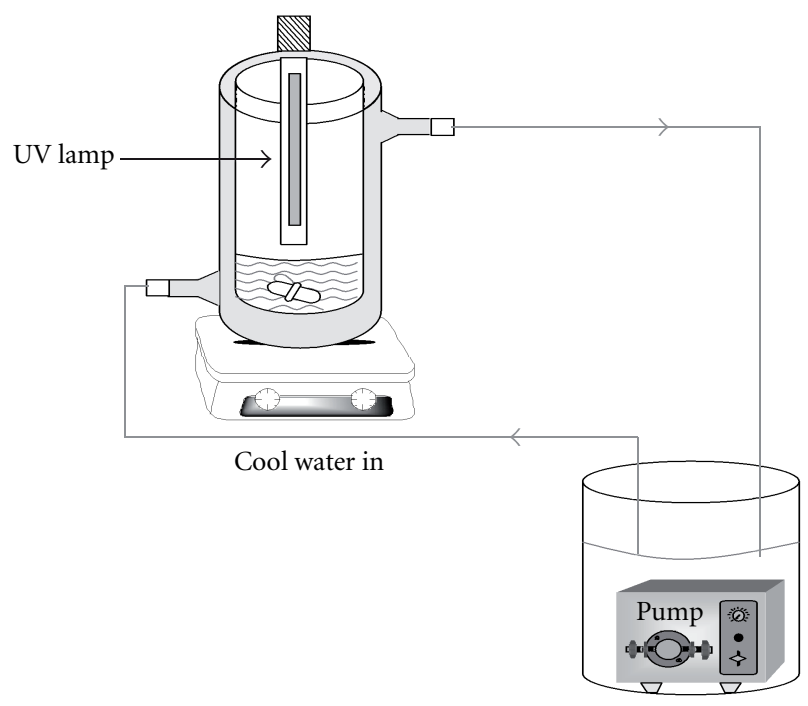

FIgURE 1: Schematic diagram for batch photoreactor.

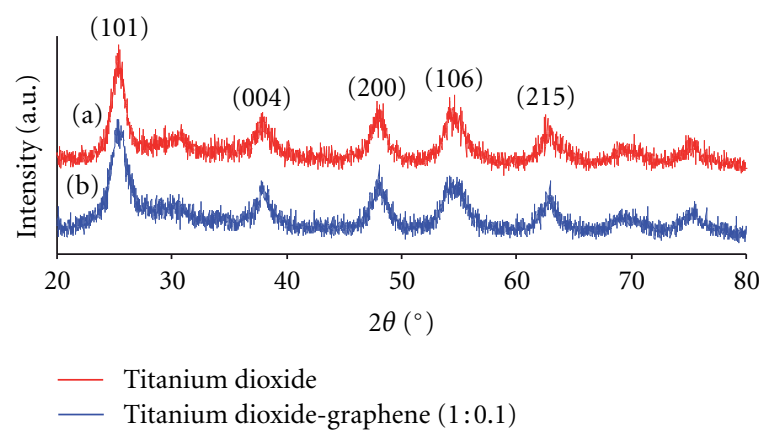

FIgURE 2: XRD spectra of (a) titanium dioxide, (b) $\mathrm{TiO}_{2}$-graphene $(1: 0.1)$.

$63.08^{\circ}$ are attributed to the anatase phase [23]. The absence of the peaks at $2 \theta=27.5^{\circ}$ and $30.8^{\circ}$ indicates that the rutile and brookite phases are not present. In addition, it is observed that the peak width broadened slightly with the introduction of graphene. It can be seen from Table 1 that the average crystallite size of $\mathrm{TiO}_{2}$ - $\mathrm{G}$ is smaller than that of pristine $\mathrm{TiO}_{2}$. It is thus inferred that due to the strong interactions between $\mathrm{TiO}_{2}$ and graphene during the hydrolysis of the sol samples, the $\mathrm{TiO}_{2}$ crystalline particles were unable to grow larger [18].

3.2. FTIR Spectroscopy. FTIR spectroscopy was used to characterize the interaction between graphene and $\mathrm{TiO}_{2}$ nanoparticles. The FTIR spectrum of graphene oxide (Figure $3(\mathrm{a}))$ shows bands which are attributed to the epoxide $\left(v_{\mathrm{C}-\mathrm{O}-\mathrm{C}}\right.$ at $\left.1250 \mathrm{~cm}^{-1}\right)$, carboxylic acid $\left(v_{\mathrm{C}=\mathrm{O}}\right.$ at $\left.1720 \mathrm{~cm}^{-1}\right)$, and hydroxyl groups $\left(v_{\mathrm{C}-\mathrm{OH}}\right.$ at $\left.1365 \mathrm{~cm}^{-1}\right)$ [24]. Conversely, it can be seen from Figure 3(c) that most of these oxygen functionalities had been removed in the synthesized $\mathrm{TiO}_{2}$ graphene composite. A new band at $\sim 1580 \mathrm{~cm}^{-1}$ which may be attributed to the skeletal vibrations of the graphene sheets [25] and a strong band at $\sim 500-700 \mathrm{~cm}^{-1}$, which is due to 

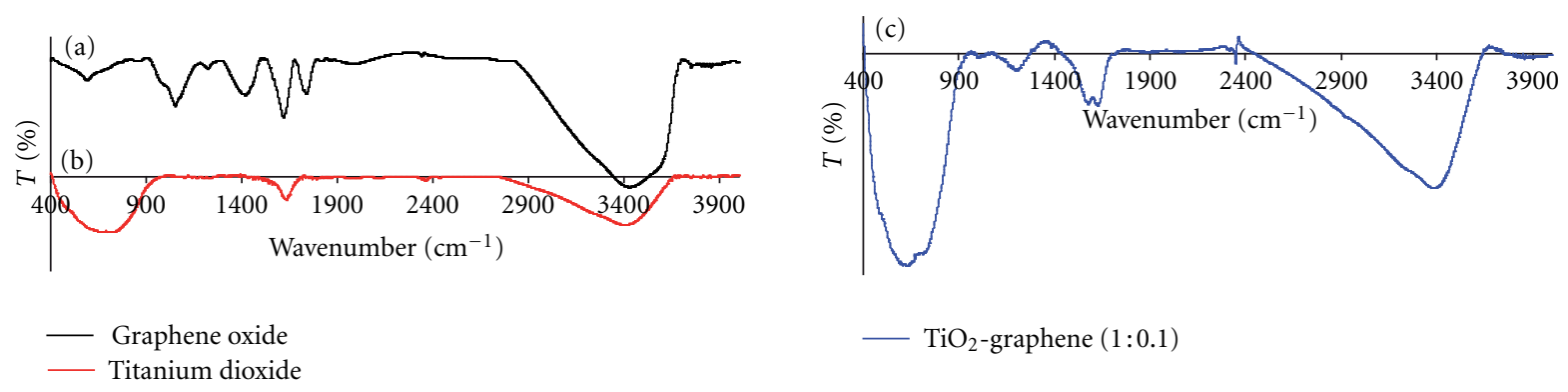

FIGURE 3: FTIR spectra of (a) acid-functionalized graphene oxide, (b) titanium dioxide, and (c) $\mathrm{TiO}_{2}$-graphene.

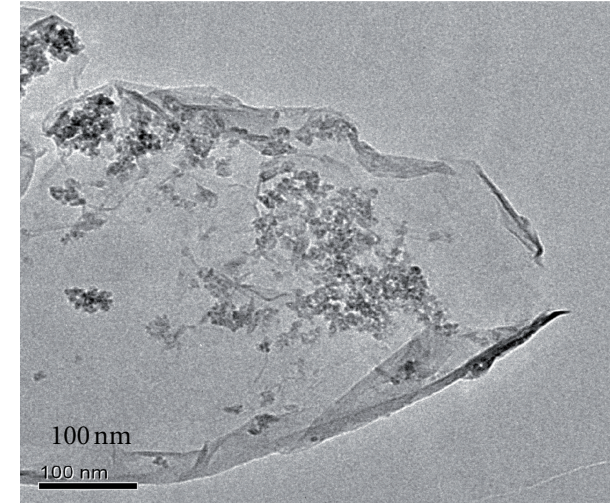

(a)

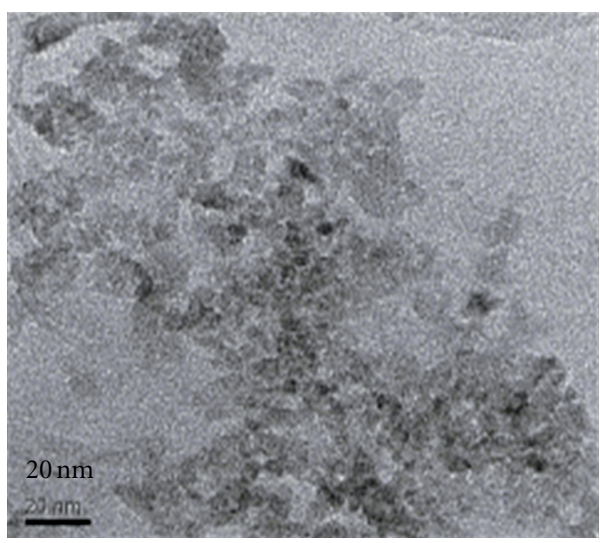

(c)

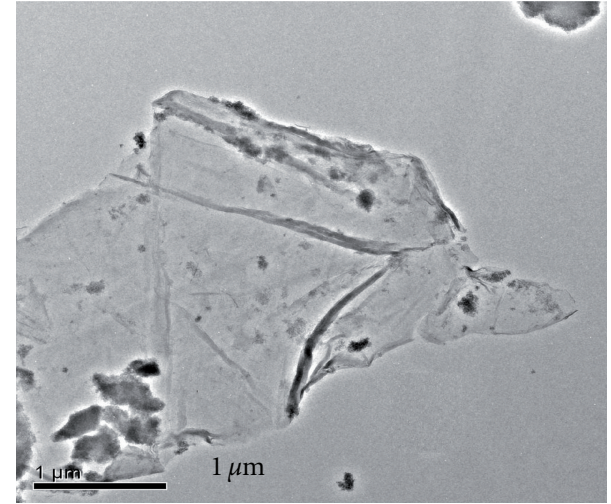

(b)

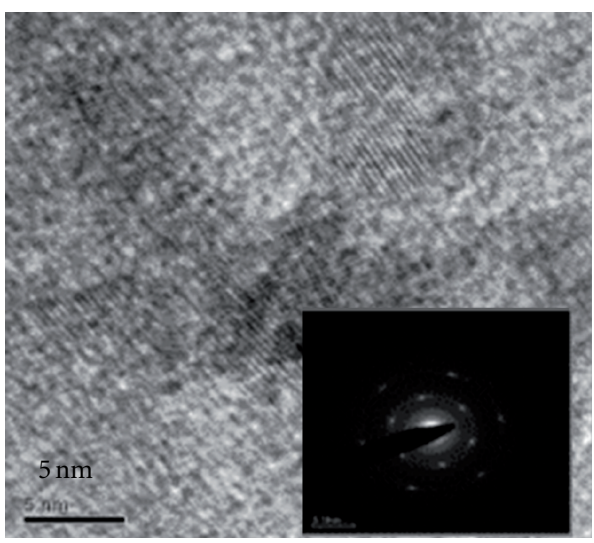

(d)

FIgURE 4: (a-c) HRTEM images of $\mathrm{TiO}_{2}$-G. (d) Crystal fringes of $\mathrm{TiO}_{2}$ with a selective area electron diffraction pattern in the inset.

the Ti-O-Ti vibration [26] confirms the presence of both graphene and $\mathrm{TiO}_{2}$ in the synthesized composite.

The IR spectrum of $\mathrm{TiO}_{2}$ shows a broad band at $\sim 3400 \mathrm{~cm}^{-1}$ and the band at $\sim 1625 \mathrm{~cm}^{-1}$ originates from the surface-adsorbed water, and this indicates the presence of $-\mathrm{OH}$ groups on the surface of titania [27]. Comparatively, the band at $\sim 2400-3400 \mathrm{~cm}^{-1}$ of $\mathrm{TiO}_{2}$-graphene is broader, thus indicating the presence of hydrogen bonding in the synthesized composite. Since hydrazine reduction introduces nitrogen groups onto graphene, the $\mathrm{OH}$ groups present on the surface of titania could form hydrogen bonds with the $\mathrm{NH}$ groups present on the graphene sheets to form the composite.
In addition, a new band at $\sim 1210 \mathrm{~cm}^{-1}$ is observed in the spectrum obtained for $\mathrm{TiO}_{2}-\mathrm{G}$, and this can be assigned to $\mathrm{Ti}-\mathrm{O}-\mathrm{C}$ vibrations [28]. Since hydrazine does not cause a full reduction of graphene oxide [20], the $\mathrm{Ti}-\mathrm{O}-\mathrm{C}$ bond may be formed when $\mathrm{OH}$ groups present in $\mathrm{TiO}_{2}$ react with residual $\mathrm{OH}$ groups present on the graphene sheets in removing water.

\subsection{Characterization of $\mathrm{TiO}_{2}-G$ by Transmission Electron} Microscopy. From the TEM images, it can be seen that the graphene sheets are coated with $\mathrm{TiO}_{2}$ nanoparticles. Figure 4 (c) illustrates the homogeneous dispersion of $\mathrm{TiO}_{2}$ nanoparticles on the graphene sheet, and it can be speculated that 


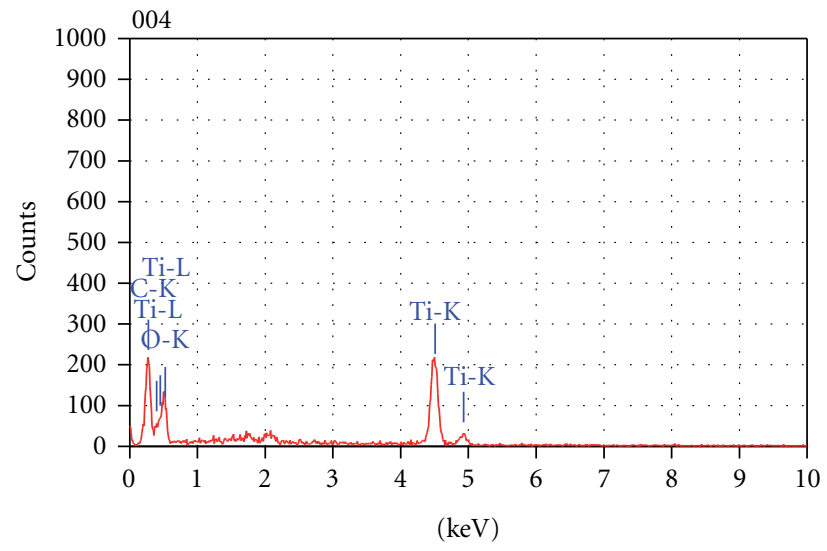

(a)

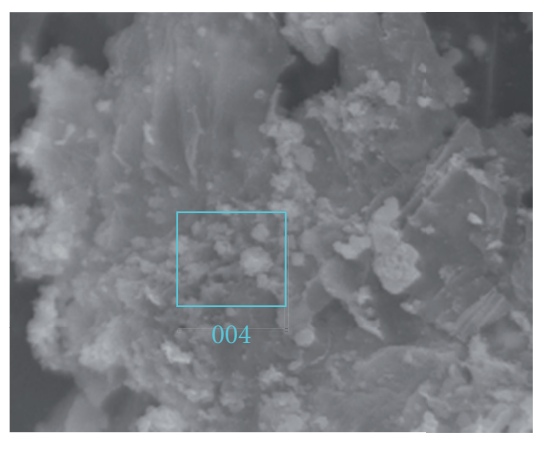

(b)

Figure 5: (a) EDX area analysis and (b) SEM micrograph for the $\mathrm{TiO}_{2}$-graphene composite.

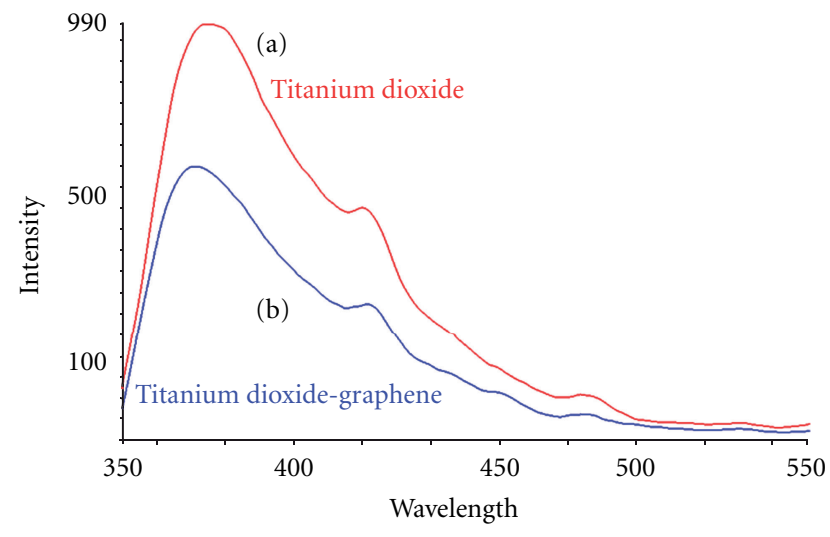

FIGURE 6: Fluorescence emission spectra of (a) titanium dioxide and (b) titanium dioxide-graphene.

TABLE 1: Structural data for the synthesized composites based on XRD spectra.

\begin{tabular}{lcc}
\hline Sample & $\begin{array}{c}\text { Average } \\
\text { crystallite } \\
\text { size }(\mathrm{nm})\end{array}$ & $\begin{array}{c}\text { Phase } \\
\text { composition }\end{array}$ \\
\hline Titanium dioxide & 7.28 & $100 \%$ anatase \\
Titanium dioxide-graphene $(1: 0.1)$ & 5.94 & $100 \%$ anatase
\end{tabular}

${ }^{\mathrm{a}}$ Determined by the use of the Debye-Scherrer equation [9] using the full width at half maximum (FHWM) for the 101 anatase XRD peak.

TABLE 2: EDX elemental microanalysis (atom \%) of $\mathrm{TiO}_{2}$-graphene sample.

\begin{tabular}{lccc}
\hline Sample & $\mathrm{C}(\%)$ & $\mathrm{O}(\%)$ & $\mathrm{Ti}(\%)$ \\
\hline Titanium dioxide-graphene $(1: 0.1)$ & 40.22 & 42.48 & 17.30 \\
\hline
\end{tabular}

under ultrasonic irradiation, the graphene sheets interact with $\mathrm{TiO}_{2}$ through strong chemisorption and physisorption [29]. In addition, the graphene sheets appear transparent and are folded over one edge [24]. Thus, this indicates that single graphene sheets have been isolated.

3.4. Energy Dispersive X-Ray Elemental Microanalysis. The EDX spectrum shows the presence of $\mathrm{Ti}, \mathrm{C}$, and $\mathrm{O}$ atoms in the synthesized $\mathrm{TiO}_{2}$-graphene composite (Figure 5). It can be seen from Table 2 that the atom percentage of $\mathrm{O}$ is slightly more than twice that of Ti. This indicated that some oxygen functionalities remained on the graphene sheets after hydrazine reduction, which is in agreement with the IR spectrum obtained.

3.5. Fluorescence Emission Spectroscopy. Since photocatalytic activity is a function of lifetime and trapping of electron and hole pairs, fluorescence emission spectra are a useful characterization technique to investigate the efficiency of charge carrier trapping and to understand the fate of $\mathrm{e}-\mathrm{h}+$ pairs in semiconductor particles like $\mathrm{TiO}_{2}$ [21]. Recombination of electron and hole pairs can emit energy in the form of fluorescence [30], and as seen from Figure 6, the fluorescence intensity of $\mathrm{TiO}_{2}-\mathrm{G}$ is lower than that of $\mathrm{TiO}_{2}$. This indicates that the recombination of electron and hole pairs is suppressed in the presence of graphene, which possibly indicates an increase in photocatalytic efficiency. In addition, the fluorescence emission spectra displayed 3 main peaks at $380 \mathrm{~nm}, 420 \mathrm{~nm}$, and $484 \mathrm{~nm}$ for both $\mathrm{TiO}_{2}$ and $\mathrm{TiO}_{2}-\mathrm{G}$, which are attributed to the self-trapped excitons localized on $\mathrm{TiO}_{6}$ octahedra and oxygen vacancies [31]. Thus, this indicates that the presence of graphene does not alter the mechanism of $\mathrm{TiO}_{2}$ photocatalysis.

3.6. Method Validation. Due to the polarity of APs, derivatization of the analytes is required prior to GC-FID analyses [32]. Silylation using bis(trimethylsilyl)trifluoroacetamide (BSTFA) is a rapid and commonly used derivatization technique in which the active hydrogen of the hydroxyl groups is converted into trimethylsilyl (TMS) group [33]. Thermally stable and highly volatile derivatives are obtained, and this results in improved gas chromatographic parameters 
TABLE 3: Quantitative data: linearity, precision, $\operatorname{LOD}(\mathrm{S} / N=3)$, and LOQ $(\mathrm{S} / N=10)$.

\begin{tabular}{|c|c|c|c|c|}
\hline Analyte & Correlation coefficient $^{\mathrm{a}}$ & $\% \operatorname{RSD}(n=3)$ & LOD $(\mu \mathrm{g} / \mathrm{L})$ & LOQ $(\mu \mathrm{g} / \mathrm{L})$ \\
\hline 4-n-Heptylphenol & 0.99981 & 9.98 & 0.73 & 2.44 \\
\hline 4-n-Octylphenol & 0.99561 & 11.19 & 0.77 & 2.56 \\
\hline 4-Nonylphenol & 0.98659 & 11.51 & 0.38 & 1.26 \\
\hline
\end{tabular}

${ }^{\mathrm{a}}$ Linear range: $5-1000 \mu \mathrm{g} / \mathrm{L}$.

TABLE 4: Percentage of degradation of alkylphenols after UV irradiation for 3 hours with the different photocatalysts.

\begin{tabular}{lccc}
\hline Photocatalyst & 4-n-Heptylphenol & 4-Nonylphenol & 4-n-Octylphenol \\
\hline $\mathrm{P} 25$ commercial photocatalyst & 60 & 20 & 30 \\
$\mathrm{TiO}_{2}-\mathrm{G}(1: 0.1)$ & 65 & 40 & 50 \\
$\mathrm{TiO}_{2}-\mathrm{G}(1: 0.2)$ & 80 & 38 & 45 \\
Synthesized $\mathrm{TiO}_{2}$ & 40 & 30 & 40 \\
\hline
\end{tabular}

such as accuracy, reproducibility, sensitivity, and resolution [34]. Liquid-liquid extraction was employed to monitor the degradation rates since it is a simple, fast, and reliable technique for monitoring the disappearance of the parent compound [35]. In order to access the practicality and suitability of this proposed LLE method, the optimized extraction conditions were used to determine the extraction method's repeatability, linearity, limits of detection (LOD), and limits of quantification (LOQ). The results are summarized in Table 3. Repeatability was evaluated by triplicate analysis at the various analyte concentrations within the linear range of the extraction method. Satisfactory repeatability of relative standard deviations (RSDs) 9 to $12 \%$ was obtained. The linearity of this extraction method was evaluated at five different concentrations, ranging from 5 to $1000 \mu \mathrm{g} / \mathrm{L}$. The limit of detection of the analytes was determined (between 0.4 and $0.8 \mu \mathrm{g} / \mathrm{L}$ ) based on $S / N$ ratio is 3 , while the limit of quantification (LOQ) was calculated (between 1.3 and $2.6 \mu \mathrm{g} / \mathrm{L}$ ) based on the definition of $S / N=10$.

3.6.1. Comparison of Photocatalytic Efficiency of the Synthesized $\mathrm{TiO}_{2}-\mathrm{G}, \mathrm{TiO}_{2}$, and Commercial Photocatalyst P25 on Alkylphenols in Wastewater Samples. Prior photocatalysis, wastewater samples were collected from local drainages and extracted using optimized LLE method. No target compounds were detected in the wastewater samples. Therefore, experiments were designed with spiked $(100 \mu \mathrm{g} / \mathrm{L}$ of alkylphenols) wastewater samples. The degradation profiles of alkylphenols are presented in Figure 7. The concentrations of photocatalyst used for each experiment is $0.1 \mathrm{~g} / \mathrm{L}$. A low dosage of photocatalyst loading was chosen for the comparison of photocatalytic efficiency since it would be more cost effective for practical applications. In order to confirm that the source of decrease in analyte response was due to photodegradation rather than sample loss from extraction, a control experiment was carried out in which the sample solution was continuously stirred over a period of 3 hours and the variation in concentration was monitored. It can be seen that the percentage of the original analyte left remained unchanged. Thus photodegradation and not sample loss was the main source of declining peak areas which was observed in the subsequent experiments.

In addition, to monitor the effects due to direct photolysis only, the sample solution was irradiated with UV light. It was observed that the concentrations decreased slightly with UV irradiation, with more than $90 \%$ of the original solution still remaining at the end of 3 hours.

When the photocatalysts were added, the peak areas of the analytes declined significantly, thus indicating the efficacy of advanced oxidation processes for the removal of these phenolic endocrine disrupting compounds. Figures 8(a) and 8 (b) show the chromatogram after the dark adsorption period $\left(C_{0}\right)$ and the chromatogram after the addition of $\mathrm{TiO}_{2}-\mathrm{G}$ photocatalyst with UV irradiation for 3 hours, respectively. The percentage of individual analytes that remained after 3 hours of UV irradiation with the different photocatalysts is shown in Table 4. It was observed that $\mathrm{TiO}_{2}$-graphene composite with $\mathrm{Ti}: \mathrm{C}$ ratio of $1: 0.1$ exhibited enhanced photocatalytic efficiencies for the photodegradation of the analytes over that of pristine $\mathrm{TiO}_{2}$, which was synthesized in the same way. As a comparison, the photocatalytic efficiencies were compared to that of the commercial $\mathrm{TiO}_{2}$ photocatalyst, which has a phase composition of $80 \%$ anatase and $20 \%$ rutile. The synthesized $\mathrm{TiO}_{2}$-graphene $(1: 0.1)$ also displayed higher photocatalytic efficiency as compared to that of the commercial $\mathrm{TiO}_{2}$ photocatalyst P25 for the degradation of 4-nonylphenol and 4-n-octylphenol. On the other hand, the $\mathrm{TiO}_{2}$-graphene photocatalyst with a higher carbon loading (Ti : C ratio of $1: 0.2$ ) had the highest photocatalytic efficiency for the photodegradation of 4 -n-heptylphenol. The degradation rate of 4-nonylphenol was the lowest since technical nonylphenol is a mixture of isomers which contain both straight- and branched-chain NPs [36].

3.6.2. Performance of $\mathrm{TiO}_{2}-\mathrm{G}$ Composite on Photocatalytic Efficiency. From the fluorescence emission spectra obtained, it can be seen that the presence of graphene can suppress the recombination of electron and hole pairs of $\mathrm{TiO}_{2}$. Under UV irradiation, electrons are excited from the valence band (VB) to the conduction band (CB) of the anatase, thus creating a hole in the valence band. In the absence of 


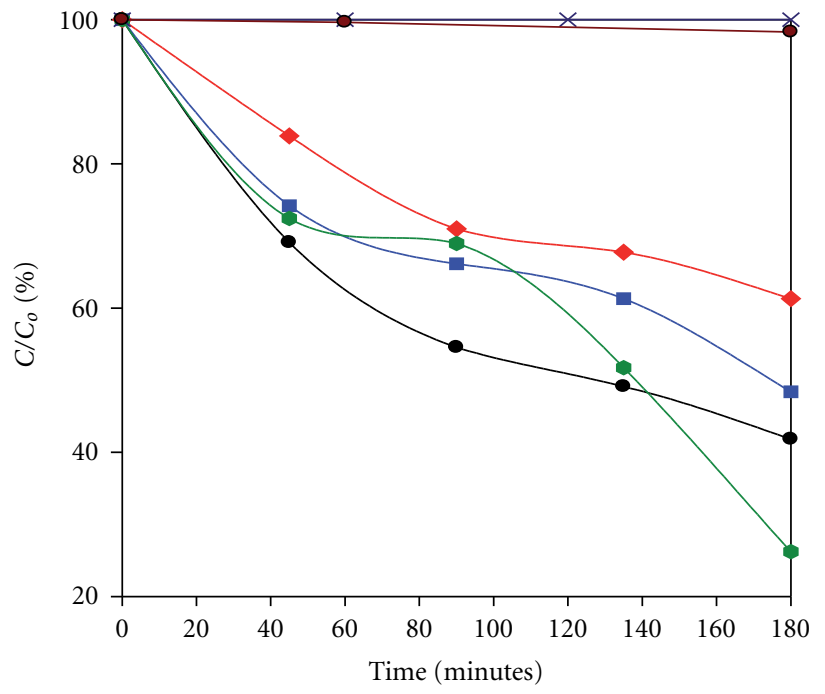

(a)

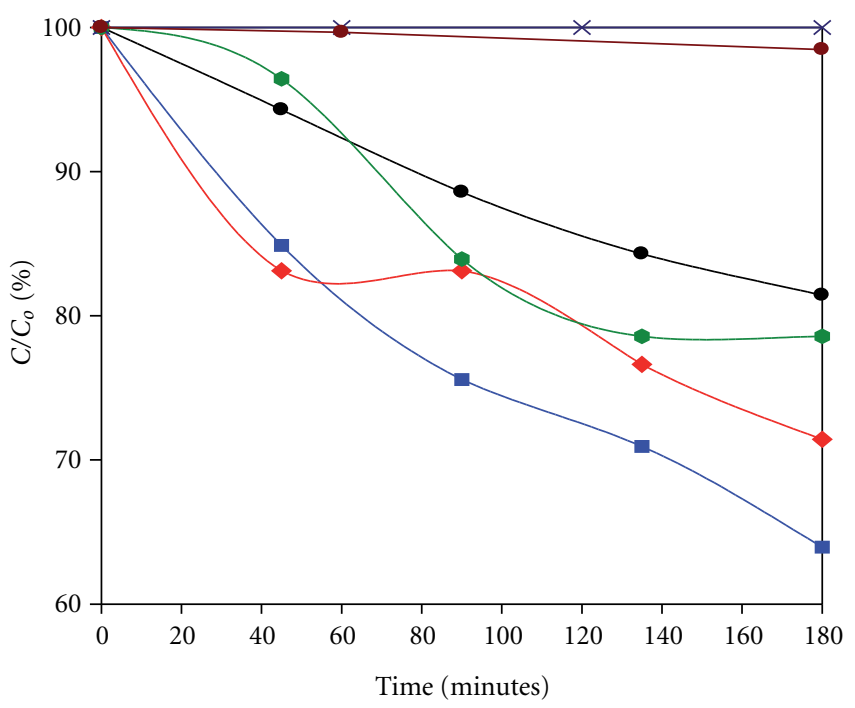

(b)

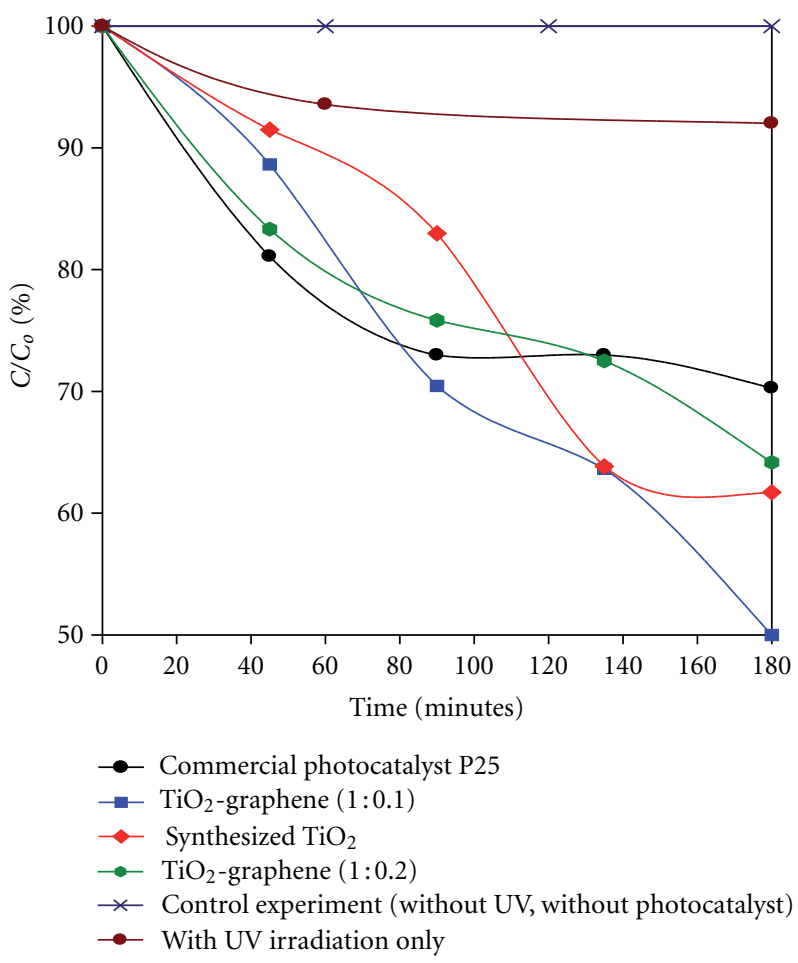

(c)

Figure 7: Photocatalytic degradation profiles of (a) 4-n-heptylphenol, (b) 4-nonylphenol, and (c) 4-n-octylphenol; $C / C_{0}$ refers to the percentage of original amount remaining.

graphene, most of these charges recombine and only a small fraction participate in photocatalytic reactions $(<1 \%)$, thus resulting in low photoefficiency. Given the semiconducting and unique electronic properties of graphene, electrons from the anatase particles can be transferred to the graphene sheets, thus allowing charge separation, stabilization, and hindered recombination [37]. Consequently, the holes on the anatase particles are longer-lived and thus the $\mathrm{TiO}_{2}-\mathrm{G}$ composite has higher photocatalytic efficiency.
In addition, the large specific surface area of graphene sheets can also account for the enhanced photocatalytic efficiency of $\mathrm{TiO}_{2}-\mathrm{G}$. Graphene sheets can adsorb the alkylphenol molecules in aqueous solutions and concentrate them on the surface of titania [38]. It was noted that the APs at time $=0 \mathrm{~min}$ are largely decreased from the prepared initial concentration due to strong adsorption on the photocatalyst surface [32]. Given the highly hydrophobic nature of the long-chained alkylphenols, they tend to adsorb on the highly 


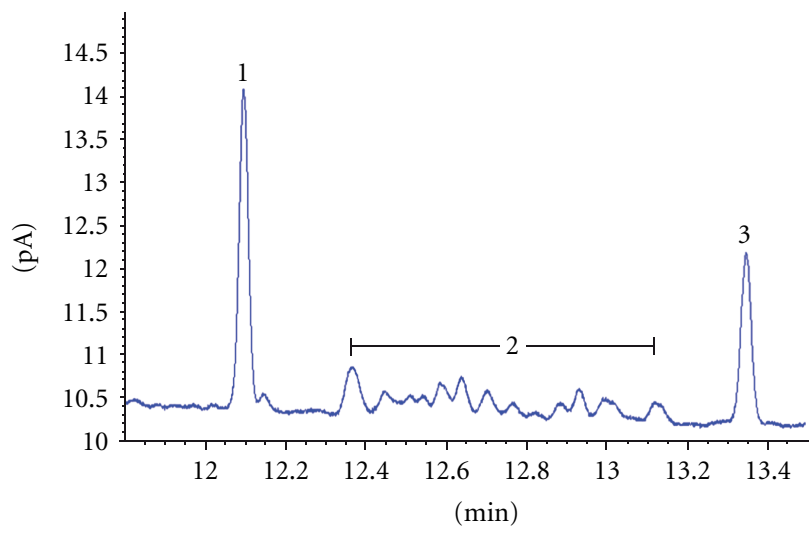

(a)

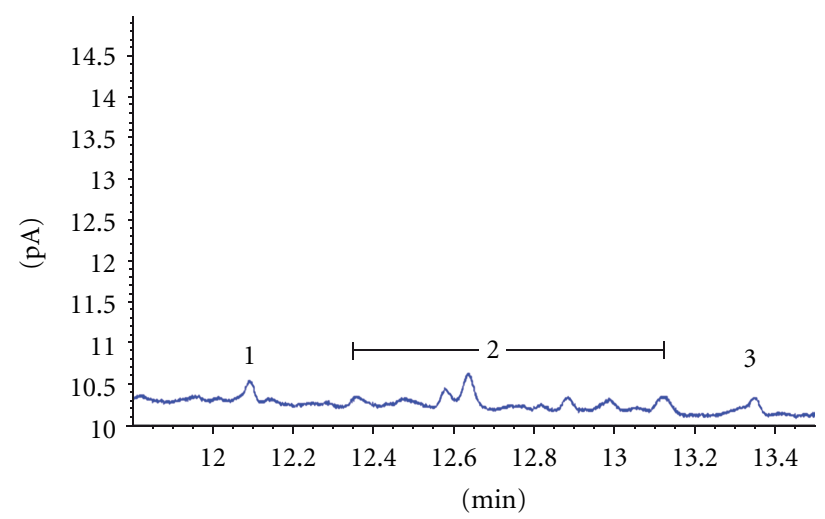

(b)

FIGURE 8: Extracted chromatograms wastewater (a) after 3 hours exposure of UV radiation without catalyst and (b) after addition of TiO ${ }_{2}$ graphene composite (1:0.1 ratio) with UV exposure for 3 hours. Peak identification: (1) 4-n-heptylphenol, (2) 4-nonylphenol (mixture of isomers), (3) 4-n-octylphenol.

hydrophobic graphene sheets. As a result, the dispersion of $\mathrm{TiO}_{2}$ on graphene sheets and adsorption of the alkylphenol molecules created many active sites for photocatalytic degradation and thus enhanced the photocatalytic efficiency.

The photocatalytic efficiency of the $\mathrm{TiO}_{2}-\mathrm{G}$ composite would increase with increase of the graphene percentage at optimum experimental conditions. The $\mathrm{TiO}_{2}-\mathrm{G}$ composite with $\mathrm{Ti}$ : $\mathrm{C}$ mass ratio of $1: 0.1$ displayed highest photocatalytic efficiency for the degradation of 4-nonylphenol and 4-n-octylphenol. One reason for the reduced photocatalytic efficiency with increase of graphene content is that higher composition of graphene sheets can shield the UV light from absorption by $\mathrm{TiO}_{2}$ nanoparticles [18]. Thus, with reduced UV adsorption by the $\mathrm{TiO}_{2}$ particles, the composite would have reduced photocatalytic efficiency. Further studies are required to investigate this mechanism. From the experimental results obtained, higher photocatalytic efficiencies can be achieved with a graphene composition which is only $10 \%$ that of $\mathrm{TiO}_{2}$ present for 4-n-nonylphenol and 4-octylphenol whereas a higher graphene loading $(20 \%$ of $\mathrm{TiO}_{2}$ present) in the synthesized composite was favorable for photodegradation of 4-heptylphenol. Thus, the presence of a small percentage of graphene can have synergetic effects on the photocatalytic efficiency of $\mathrm{TiO}_{2}$.

\section{Conclusion}

In this study, $\mathrm{TiO}_{2}$-graphene composite has been successfully synthesized and characterized. Compared to that of pristine $\mathrm{TiO}_{2}, \mathrm{TiO}_{2}-$ Graphene exhibited enhanced photocatalytic efficiency of alkylphenols. By varying the composition of graphene in the photocatalyst, the photocatalytic efficiency was also varied. The photocatalytic efficiency of the photocatalyst exhibited better photocatalytic efficiency than the commercial $\mathrm{TiO}_{2}$ photocatalyst, P25 with optimal loading of graphene. This might be due to the following reasons: (i) large surface area of graphene allowed it to act as a coadsorbent for the alkylphenol molecules and it resulted in more active sites for photocatalytic reactions to occur and (ii) graphene could inhibit the recombination of electron and hole pairs of $\mathrm{TiO}_{2}$, thus enhancing charge transfer and allowing the oxidation of adsorbed molecules.

\section{Acknowledgments}

The author gratefully acknowledges the support of this research by the National University of Singapore and also would like to acknowledge the support provided by the King Abdul Aziz City for Science and Technology through the Science and Technology Unit at the King Fahd University of Petroleum and Minerals for funding this work through Project no. 10-WAT1396-04 as part of the National Science Technology and Innovation Plan.

\section{References}

[1] C. Basheer, H. K. Lee, and K. S. Tan, "Endocrine disrupting alkylphenols and bisphenol-A in coastal waters and supermarket seafood from Singapore," Marine Pollution Bulletin, vol. 48, pp. 1145-1167, 2004.

[2] S. Yamazaki, T. Mori, T. Katou, M. Sugihara, A. Saeki, and T. Tanimura, "Photocatalytic degradation of 4-tert-octylphenol in water and the effect of peroxydisulfate as additives," Journal of Photochemistry and Photobiology A, vol. 199, no. 2-3, pp. 330-335, 2008.

[3] European Commission, "Directive 2000/60/EC of the European Parliament and of the Council of 23 October 2000 establishing a framework for Community action in the field of water policy," Official Journal, vol. L 327, pp. 0001-0073, 2000.

[4] I. Gültekin and N. H. Ince, "Synthetic endocrine disruptors in the environment and water remediation by advanced oxidation processes," Journal of Environmental Management, vol. 85, no. 4, pp. 816-832, 2007.

[5] R. Loos, J. Wollgast, J. Castro-Jiménez et al., "Laboratory intercomparison study for the analysis of nonylphenol and octylphenol in river water," Trends in Analytical Chemistry, vol. 27, no. 1, pp. 89-95, 2008. 
[6] S. S. Watson, D. Beydoun, J. A. Scott, and R. Amal, "The effect of preparation method on the photoactivity of crystalline titanium dioxide particles," Chemical Engineering Journal, vol. 95, no. 1, pp. 213-220, 2003.

[7] S. Kohtani, S. Makino, A. Kudo, K. Tokumura, and Y. Ishigaki, "Photocatalytic degradation of 4-n-nonylphenol under irradiation from solar simulator: comparison between $\mathrm{BiVO}_{4}$ and $\mathrm{TiO}_{2}$ photocatalysts," Chemistry Letters, no. 7, pp. 660-661, 2002.

[8] B. Tryba, "Increase of the photocatalytic activity of $\mathrm{TiO}_{2}$ by carbon and iron modifications," International Journal of Photoenergy, vol. 2008, Article ID 721824, 15 pages, 2008.

[9] D. Li, M. B. Müller, S. Gilje, R. B. Kaner, and G. G. Wallace, "Processable aqueous dispersions of graphene nanosheets," Nature Nanotechnology, vol. 3, no. 2, pp. 101-105, 2008.

[10] S. Kwon, M. Fan, A. T. Cooper, and H. Yang, "Photocatalytic applications of micro- and $\mathrm{Nano}^{-\mathrm{TiO}_{2}}$ in environmental engineering," Critical Reviews in Environmental Science and Technology, vol. 38, no. 3, pp. 197-226, 2008.

[11] Y. Yu, J. C. Yu, C. Y. Chan et al., "Enhancement of adsorption and photocatalytic activity of $\mathrm{TiO}_{2}$ by using carbon nanotubes for the treatment of azo dye," Applied Catalysis B, vol. 61, no. 1-2, pp. 1-11, 2005.

[12] S. H. Xie, J. Y. Li, Y. Qiao et al., "Multiferroic $\mathrm{CoFe}_{2} \mathrm{O}_{4}-\mathrm{Pb}\left(\mathrm{Zr}_{0.52} \mathrm{Ti}_{0.48}\right) \mathrm{O}_{3}$ nanofibers by electrospinning," Applied Physics Letters, vol. 92, no. 6, Article ID 062901, 3 pages, 2008.

[13] G. Eda, G. Fanchini, and M. Chhowalla, "Large-area ultrathin films of reduced graphene oxide as a transparent and flexible electronic material," Nature Nanotechnology, vol. 3, no. 5, pp. 270-274, 2008.

[14] A. K. Geim and K. S. Novoselov, "The rise of graphene," Nature Materials, vol. 6, no. 3, pp. 183-191, 2007.

[15] S. Stankovich, D. A. Dikin, R. D. Piner et al., "Synthesis of graphene-based nanosheets via chemical reduction of exfoliated graphite oxide," Carbon, vol. 45, no. 7, pp. 1558-1565, 2007.

[16] Y. Zhang, N. Zhang, Z. R. Tang, and Y. J. Xu, "Improving the photocatalytic performance of graphene- $\mathrm{TiO}_{2}$ nanocomposites via a combined strategy of decreasing defects of graphene and increasing interfacial contact," Physical Chemistry Chemical Physics, vol. 14, no. 25, pp. 9167-9175, 2012.

[17] N. Zhang, Y. Zhang, X. Pan, X. Fu, S. Liu, and Y. J. Xu, "Assembly of CdS nanoparticles on the two-dimensional graphene scaffold as visible-light-driven photocatalyst for selective organic transformation under ambient conditions," The Journal of Physical Chemistry C, vol. 115, no. 47, pp. 23501-23511, 2011.

[18] Y. Zhang, Z. R. Tang, X. Fu, and Y. J. Xu, "Engineering the unique $2 \mathrm{D}$ mat of graphene to achieve graphene- $\mathrm{TiO}_{2}$ nanocomposite for photocatalytic selective transformation: what advantage does graphene have over its forebear carbon nanotube?" ACS Nano, vol. 5, no. 9, pp. 7426-7435, 2011.

[19] Y. Zhang, Z. R. Tang, X. Z. Fu, and Y. J. Xu, “TiO ${ }_{2}$-graphene nanocomposites for gas-phase photocatalytic degradation of volatile aromatic pollutant: is $\mathrm{TiO}_{2}$-graphene truly different from other $\mathrm{TiO}_{2}$-carbon composite materials?" ACS Nano, vol. 4, no. 12, pp. 7303-7314, 2010.

[20] W. S. Hummers and R. E. Offeman, "Preparation of graphitic oxide," Journal of the American Chemical Society, vol. 80, no. 6, article 1339, 1958.

[21] Y. Yu, J. C. Yu, C. Y. Chan et al., "Enhancement of photocatalytic activity of mesoporous $\mathrm{TiO}_{2}$ by using carbon nanotubes," Applied Catalysis A, vol. 289, no. 2, pp. 186-196, 2005.
[22] U. I. Gaya and A. H. Abdullah, "Heterogeneous photocatalytic degradation of organic contaminants over titanium dioxide: a review of fundamentals, progress and problems," Journal of Photochemistry and Photobiology C, vol. 9, no. 1, pp. 1-12, 2008.

[23] K. Porkodi and S. D. Arokiamary, "Synthesis and spectroscopic characterization of nanostructured anatase titania: a photocatalyst," Materials Characterization, vol. 58, no. 6, pp. 495-503, 2007.

[24] Y. Si and E. T. Samulski, "Synthesis of water soluble graphene," Nano Letters, vol. 8, no. 6, pp. 1679-1682, 2008.

[25] C. Nethravathi and M. Rajamathi, "Chemically modified graphene sheets produced by the solvothermal reduction of colloidal dispersions of graphite oxide," Carbon, vol. 46, no. 14, pp. 1994-1998, 2008.

[26] J. Wang, G. Zhao, Z. H. Zhang et al., "Investigation on degradation of azo fuchsine using visible light in the presence of heat-treated anatase $\mathrm{TiO}_{2}$ powder," Dyes and Pigments, vol. 75, no. 2, pp. 335-343, 2007.

[27] G. Liu, X. Wang, Z. Chen, H. M. Cheng, and G. Lu, “The role of crystal phase in determining photocatalytic activity of nitrogen doped $\mathrm{TiO}_{2}$," Journal of Colloid and Interface Science, vol. 329, no. 2, pp. 331-338, 2009.

[28] S. Şener, M. Erdemoğlu, M. Asiltürk, and H. Sayilkan, "The effect of silane modification on the adsorptive properties of natural pyrophyllite and synthetic titanium-based powders prepared by the sol-gel process," Turkish Journal of Chemistry, vol. 29, pp. 487-495, 2005.

[29] G. Williams, B. Seger, and P. V. Kamt, " $\mathrm{TiO}_{2}$-graphene nanocomposites. UV-assisted photocatalytic reduction of graphene oxide," ACS Nano, vol. 2, no. 7, pp. 1487-1491, 2008.

[30] H. B. Li, X. C. Duan, G. C. Liu, and L. L. Li, "Synthesis and characterization of copper ions surface-doped titanium dioxide nanotubes," Materials Research Bulletin, vol. 43, no. 8-9, pp. 1971-1981, 2008.

[31] J. Zhou, Y. Zhang, X. S. Zhao, and A. K. Ray, "Photodegradation of benzoic acid over metal-doped $\mathrm{TiO}_{2}$," Industrial and Engineering Chemistry Research, vol. 45, no. 10, pp. 3503-3511, 2006.

[32] C. Basheer and H. K. Lee, "Analysis of endocrine disrupting alkylphenols, chlorophenols and bisphenol-A using hollow fiber-protected liquid-phase microextraction coupled with injection port-derivatization gas chromatography-mass spectrometry," Journal of Chromatography A, vol. 1057, no. 1-2, pp. 163-169, 2004.

[33] Thermo Scientific, "Silylation (Analytical/Chromatography)," http://www.thermo.com/com/cda/product/detail/0, 1055, 10144049, 00.html.

[34] D. Li, J. Park, and J. R. Oh, "Silyl derivatization of alkylphenols, chlorophenols, and bisphenol A for simultaneous GC/MS determination," Analytical Chemistry, vol. 73, no. 13, pp. 3089-3095, 2001.

[35] I. K. Konstantinou and T. A. Albanis, "Photocatalytic transformation of pesticides in aqueous titanium dioxide suspensions using artificial and solar light: intermediates and degradation pathways," Applied Catalysis B, vol. 42, no. 4, pp. 319-335, 2003.

[36] S. Kohtani, J. Hiro, N. Yamamoto, A. Kudo, K. Tokumura, and R. Nakagaki, "Adsorptive and photocatalytic properties of Agloaded $\mathrm{BiVO}_{4}$ on the degradation of 4-n-alkylphenols under visible light irradiation," Catalysis Communications, vol. 6, no. 3, pp. 185-189, 2005. 
[37] Y. Yao, G. Li, S. Ciston, R. M. Lueptow, and K. A. Gray, "Photoreactive $\mathrm{TiO}_{2}$ /carbon nanotube composites: synthesis and reactivity," Environmental Science and Technology, vol. 42, no. 13, pp. 4952-4957, 2008.

[38] Y. S. Luo, J. P. Liu, X. H. Xia et al., "Fabrication and characterization of $\mathrm{TiO}_{2}$ /short MWNTs with enhanced photocatalytic activity," Materials Letters, vol. 61, no. 11-12, pp. 2467-2472, 2007. 

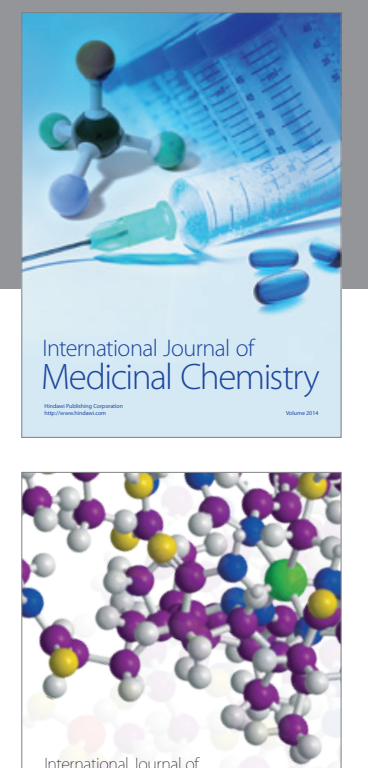

\section{Carbohydrate} Chemistry

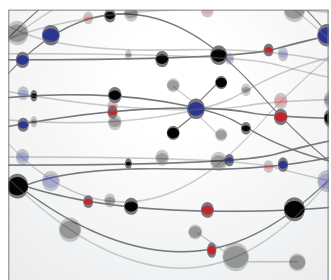

The Scientific World Journal
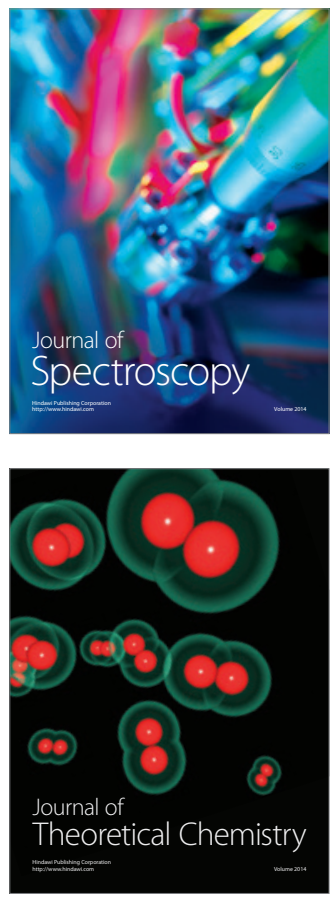
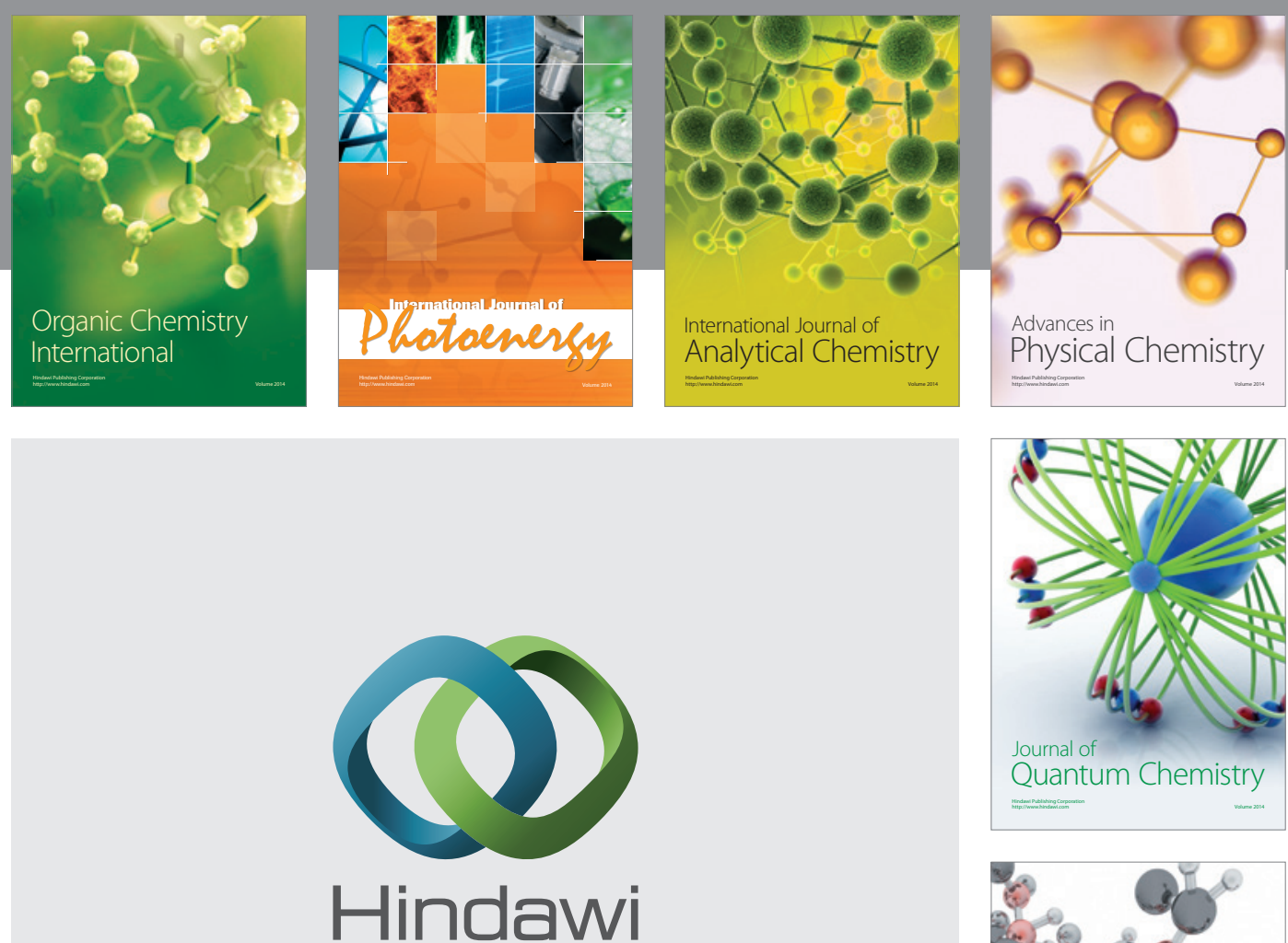

Submit your manuscripts at

http://www.hindawi.com

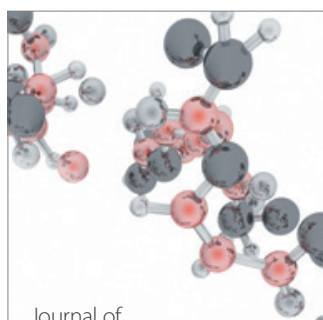

Analytical Methods

in Chemistry

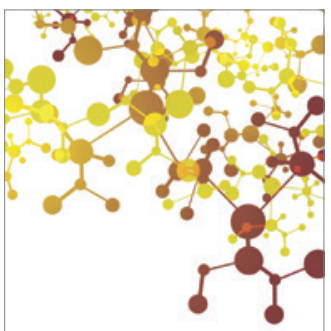

Journal of

Applied Chemistry

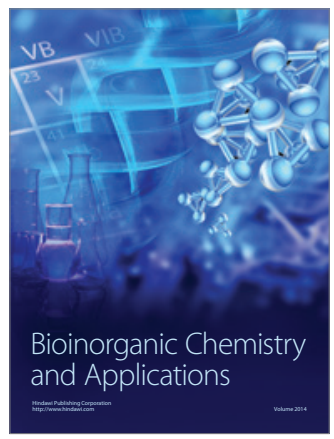

Inorganic Chemistry
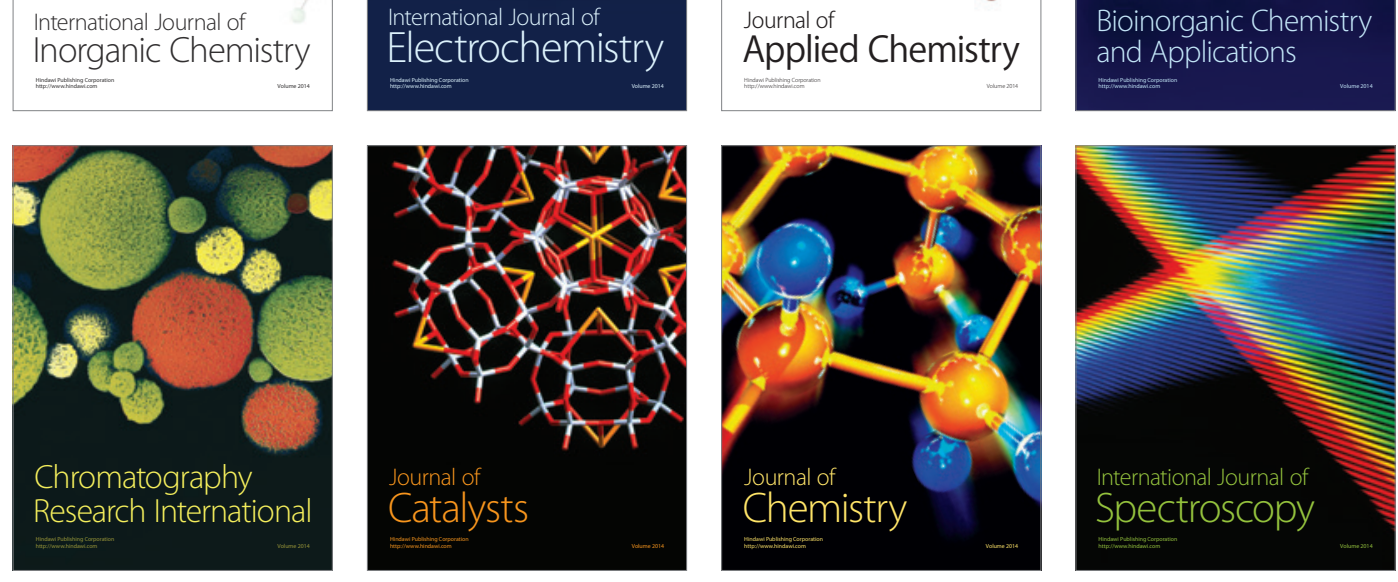